\title{
Attendance Tracking using RFID and IoT
}

\author{
Ahoud Rashid Khalfan Al \\ Tarshia \\ Nouf Salem Issa Al Sadia \\ Viswan Vimbia
}

\author{
College of Applied Science - Suhar \\ College of Applied Science - Suhar \\ College of Applied Science - Suhar
}

\begin{abstract}
Technologies played an important role in developing the way of living life in terms of making it more easer and flexible than the past. One of the technologies in prominence is RFID which stands for "Radio-Frequency Identification". RFID uses electromagnetic fields to transfer data that can automate identity and track using tags. This technology has been widely used in IT Asset Tracking, Logistics, Real Time Location Systems, Library systems and many more including Attendance Management in schools and colleges. Internet of Things (IoT) is considered as a technology that monitors the status of physical objects, capture meaningful data and communicate them through internet to software applications. The advancement in 4G and 5G technologies have accelerated the use of IoT and for instance with RFID wearable devices in collecting data wirelessly and sending them to software applications for better decision making. This project proposes a smart Attendance Tracking system using RFID and IoT for tracking attendance of students in classrooms. The project proposes to eradicate the time consuming, insecure and inefficient way of attendance tracking by calling names or signing on paper. A common way of attendance tracking with RFID is with the biometric system. However, this project proposes a protype using RFID and IoT with cloud-based applications that reduces IT support, decreases costs and builds intelligence to RFID tags. The prototype experiments with Ultra High Frequency RFID cards in association with Arduino Raspberry Pi microcontrollers and Microsoft Azure for cloud-based app and data storage. The software recognizes the student through the information on the UHF RFID smart card for recording attendance. Besides sending SMS or email to stakeholders confirming their presence in the class the prototype also uses built-in intelligence that informs about class timings, venue and cautioning late arrivals. The data obtained wirelessly from RFID cards can be analysed and provide the organization with information related measuring attendance at sessions, demographic visibility, engagement metrics, duration spent in seminars or events and understand interests and preferences. The project accentuates the role of RFID and IoT in enhancing the smart attendance system.
\end{abstract}

\section{Introduction}

The advancement of the online learning portals, with all information available online unlike the traditional method, has demotivated student learners to attend lectures in class rooms. Lethargic students, indifferences in college work, entangled in social network activities and many more play no important role in attaining the objectives of an institution and resulted in poor attendees for lectures. Consequently the educational administrators have to come up with ways that will ensure student participation like roll calls. However such endeavors are time consuming, stressful, cumbersome and inaccurate and eat up a chunk of the lecture time which otherwise could be used effectively (Longe O.O.2009).

Therefore to overcome these anomalies an efficient and robust method becomes necessary. RFID Radio Frequency Identification - is a technology that can be used in solving this problem. RFID is one of the automatic recognition technologies that use tags attached to objects to be identified. The two-way transmitter-reader called, Reader, sends signal to the tag and reads its response. RFID comprises a combination of antenna and microchip technology. Electricity is obtained when an electromagnetic induction is caused at the antenna between the reader and writer. This induced 
electricity is used to operate the microcontroller to receive or transmit data by radio wave (Dawes A.T. 2004). There are three types of RFID tags - passive, active or battery-assisted passive. The passive tags responds to radio energy transmitted by the reader whereas a battery-assisted tag has an embedded battery which is activated in the vicinity of a reader and responds subsequently. An active tag, on the contrary has an embedded battery which transmits signals periodically.

RFID system discussed in this paper is configured to have an Active reader with a passive tag. Tags operate at different frequency band and come in various forms. We use an Ultra High Frequency tag that has a range of one to twelve meters with a moderate to high data transfer speed. This allows students to seamlessly walk through gateways and classroom doors and not become necessary to swipe the card at the reader and thus saving time (Dawes A.T. 2004).

\section{Block Diagram}

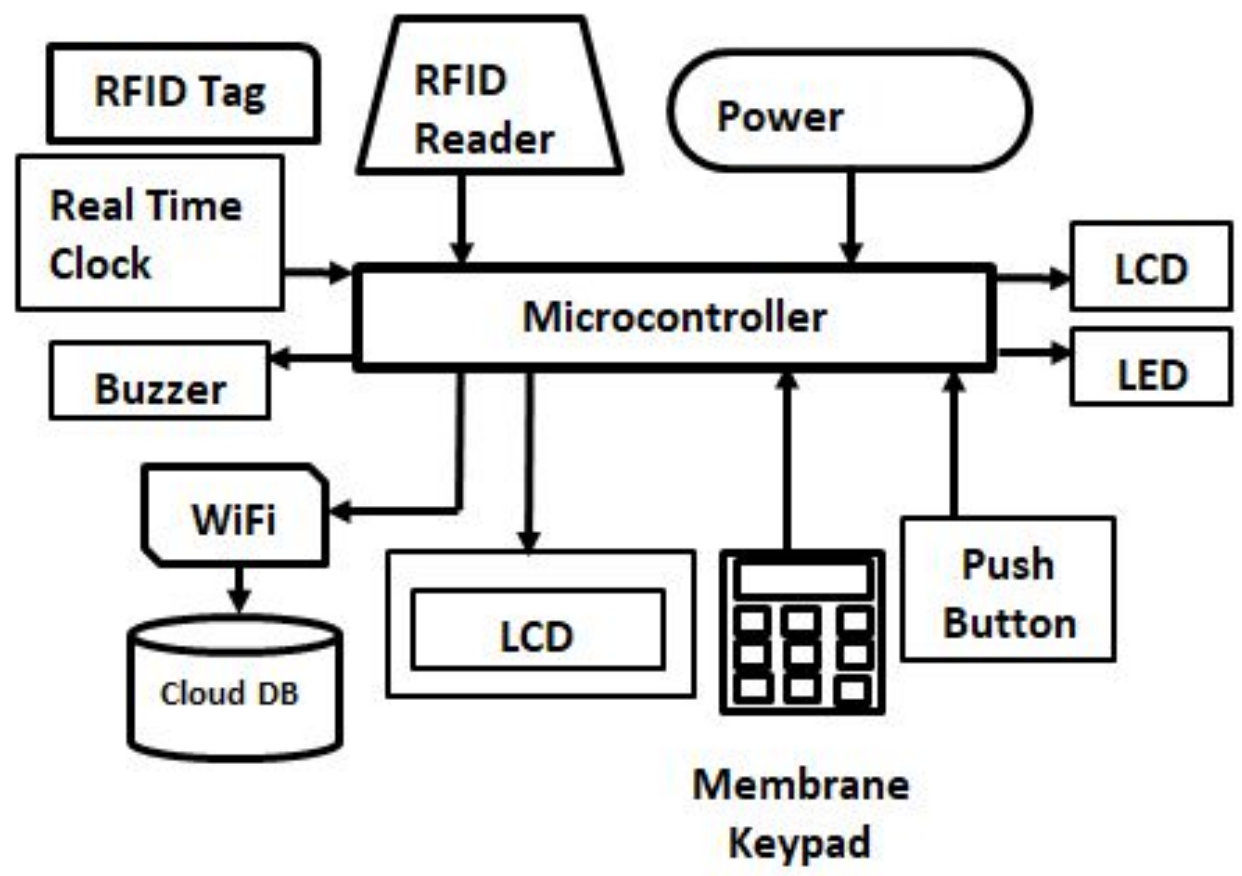

Figure 1. Block diagram of Attendance Tracking System using RFID and IoT

\section{Working}

The project presented in this paper is designed to use a single board computer - Raspberry Pi (Figure 1). On powering ON the RFID reader becomes active and reads RFID UHF tags within a range of one to twelve meters (Bardaki,C., Kourouthanassis, P. and Pramatari, K., 2012). Every card contains a unique number that identifies a student or any other employee of the institution. Every log of attendance is identified using the data of the real time clock. The software program stored in RPi compares the read number with similar data stored in database. If a match is found it checks whether it is the right time, day and venue the student wishes to enter and if found true the green led blinks ratifying the marking of attendance and recording the attendance to the database. Otherwise the red led blinks and a message is shown in the LCD display indicating denial of service. The push button is used to manually reset the system in the event of any error. The program checks for late arrivals and automatically denies recording of attendance. However, for valid reasons which governed and overruled by the lecturer, the system allows to enter attendance using the membrane keypad.

Other facilities included in the software program are to send SMS message showing the percentage 
of absence and a reminder of the scheduled classes. In the event of a workshop or seminar, the system can be set with details like date, time and venue. The workshop can be associated to groups where the system allows marking of attendance only for students or lecturers of the assigned group.

\section{Results}

In a majority of educational systems attendance is marked using roll call and manually marking on paper. The project discussed in this paper replaces this conventional method and automated the entire attendance monitoring with accuracy and speed. This system can also be used to mark attendance of employees of an organization, informing of late arrivals and many more (Nambiar A.N. 2009). The system can be further augmented to be used in car parking system marking the in/out times Mohamed A.B, Abdel- Hamid A and Mohammed K.Y. 2009).

The system prescribed in this paper is fully automated and eliminates human interaction in the process of attendance marking. Besides the LCD in the system, user interfaces are provided to be used with desktop computers and also mobile apps. This gives the benefit of viewing attendance on remote appliances. The system ensures accurate marking of attendance and avoids proxy attendances.

\section{Conclusion and Future Work}

The design of Attendance tracking using RFID and IoT discussed in this paper is intended to be used at the College of Applied Sciences, Sohar. The potential of RFID in reading data has been utilized and implemented for recording student attendance by just keeping or swiping RFID tags over the RFID reader fixed at the entrance of classrooms. The system shows success and is accepted by the lecturers of the institution. The system proves to be accurate and is effortless in operation. We will continue our search in improvising the system in tracking situations where student holds more tags which is not of their credentials and enters the lecture hall as a proxy helping fellow students in disguise.

\section{Acknowledgements}

This project is funded by the Colleges of Applied Sciences, Sohar, Oman including paper submission registration fee.

\section{References}

Longe O.O.(2009). "Implementation of Student Attendance System using RFID Technology", B. Tech Project Report, Ladoke Akintola University of Technology, Ogbomoso, Nigeria.

Dawes A.T. (2004). "Is RFID Right for Your Library", Journal of Access Services, Volume 2(4).

Bardaki,C., Kourouthanassis, P. and Pramatari, K., (2012). Deploying RFID-Enabled Services in the Retail Supply Chain: Lessons Learned toward the Internet of Things, Information Systems Management, Vol. 29.

Nambiar A.N. (2009). "A supply chain perspective of RFID Systems”, World Academy of Science, Engineering and Technology Journal, Volume 6.

Mohamed A.B, Abdel- Hamid A and Mohammed K.Y. (2009). "Implementation of an Improved secure system detection for E passport by using EPC RFID tags", World Academy of Science, Engineering and Technology Journal, Volume 6. 
